\title{
XIII. Experimental proof that the resistance to galvanic conduction is dependent on the motion of the conductor
}

\section{E. Edlund}

To cite this article: E. Edlund (1876) XIII. Experimental proof that the resistance to galvanic conduction is dependent on the motion of the conductor , Philosophical Magazine Series 5, 1:2, 89-108, DOI: $10.1080 / 14786447608639010$

To link to this article: http://dx.doi.org/10.1080/14786447608639010

曲 Published online: 13 May 2009.

Submit your article to this journal $[\pi$

Џll Article views: 2

Q View related articles $\longleftarrow$ 
LONDON, EDINBURGH, AND DUBLIN

\title{
PHILOSOPHICAL MAGAZINE
}

\author{
AND \\ JOURNAL OF SCIENCE.
}

[FIFTH SERIES.]

FEBRUARY 1876.

XIII. Experimental Proof that the Resistance to Galvanic Conduction is dependent on the Motion of the Conductor. By E. EdLUND*.

I.

TN my memoir entitled "Théorio des Phénomènes Electriques " $\uparrow \mathrm{I}$ have endeavoured to prove, in the theoretical way, that the resistance to galvanic conduction must be proportional to the intensity of the current. This conclusion from theory contradicts the view, hitherto generally received, that the resistance to conduction is independent of the currentintensity. Nevertheless, on a closer investigation of the relations, we discover that the experiments which have been instituted do not by any means justify such an assumption ; for I have shown, in the memoir just mentioned, that the various methods hitherto employed for measuring the resistance leave it in reality quite undecided whether the resistanee is or is not dependent on the intensity of the current $\ddagger$. I have therefore instituted the following experiments for the purpose of solving the question experimentally.

If $i$ signifies the current-intensity, $a$ the cross section of the conductor, $\delta$ the mass of free æther in the unit of volume of the conductor (which mass, when a galvanic current is in question, may be regarded as invariable), and $h$ the velocity of the

* Translated from a separate impression, communicated by the Author, from Poggendorff's Annalen, vol. clvi. pp. 251-278.

$\dagger K$. Svenska Vetenskaps Akademiens Handlingar. Stockholm: Norstedt et Söner. Leipzig : F. A. Brockhaus.

$\ddagger$ Op. cit. p. 25.

Phil. Mag. S. 5. Vol. 1. No. 2. Feb. 1876. 
æether, we have by the theory $i=\delta a h$. Thus the currentintensity is measured by the mass of æther which flows through the cross section of the conductor in the unit of time : this I. have called the mechanical measure of the intensity*. If $r_{0}$ denotes the resistance of the unit of length of the conductor when a current of unit intensity flows through it, then is $r_{0}$ the resistance in the usual signification of the term; and this resistance, in order to distinguish it from those which take place with other current-intensities, I have named the principal resistance to conduction $\dagger$. Consequently, if $r$ denotes the resistance with the current-intensity $i$, we have, according to the theory, $r=r_{0} i$, or, eliminating $i, r=r_{0} \delta a h$.

Theoretically, therefore, the resistance $r$ is proportional to the relative velocity between the wer molecules and the molecules of the conductor. If, then, the conductor receive a velocity $h_{1}$ in the same direction in which the æther flows, the resistance $r$ will be diminished; for it will become

$$
r=r_{0} \delta a\left(h-h_{1}\right) \text {. }
$$

If, on the contrary, we impart to the conductor the same velocity $h_{1}$, but in the opposite direction, the resistance is augmented and we get

$$
r=r_{0} \delta a\left(h+h_{1}\right) \text {. }
$$

After the current-intensity has become constant, the electromotive force of the series must, according to the theory, be equal to the sum of all the resistances. If, then, the electromotive force and the principal resistance of the battery be denoted respectively by $\mathrm{E}$ and $R_{0}$, and if $r_{0}$ signifies the principal resistance in the conductor which connects the poles of the battery, and $i$ the intensity of the current, then is $\mathrm{E}=\mathrm{R}_{0} i+r_{0} i$, whence

$$
i=\frac{\mathrm{E}}{\mathrm{R}_{0}+r_{0}} \text {. }
$$

If now to the conductor whose principal resistance is $r_{0}$ a velocity $\pm h$ is imparted, then, in accordance with what was said above, if $i_{1}$ signifies the current-intensity, it makes

whence

$$
\mathbf{E}=\mathbf{R}_{0} i_{1}+r_{0}\left(i_{1} \mp \delta a h\right),
$$

$$
i_{1}=\frac{\mathrm{E} \pm r_{0} \delta a h_{1}}{\mathrm{R}_{0}+r_{0}} \text {. }
$$

Consequently the difference of the two currents becomes

$$
i-i_{1}=: \frac{F_{0} \delta 02 h_{1}}{R_{0}+r_{0}} \cdot \text {. . . . . . }
$$

* Theorie des Phénomènes Electriques, p. 10.

† Ibid. p. 24. 
Therefore the change produced in the current-intensity by putting the conductor in motion is proportional to the velocity $h_{1}$ and the cross section $a$ of the conductor. When the resistm ance $R_{0}$ is so inconsiderable that it may be neglected in comparison with $r_{0}$, the alteration of the current-intensity is independent of the resistance.

At the first glance it might be supposed that the velocity $h_{1}$ which with accessible means can be imparted to the conductor would be so slight, in comparison with the velocity $h$ of the æther, that the alteration in question (of the current-intensity) could not be observed even with the most delicate galvanometer. The explanation, however, given in the above-mentioned memoir (p. 56), of Quincke's diaphragm-currents, intimates that the observations must be practicable; and on this account I resolved to test the theoretical result by experiment.

\section{II.}

Special mechanical arrangements would be required if we would give to solid conductors the velocity necessary for these experiments. I therefore employed liquids, which ean with facility be set in motion of sufficiently great velocity. But here two inconveniences appear, the injurious effects of which must be carefully avoided : one of them is the polarization of the plates by which the current is conducted into and out of the liquid; the other consists in the difficulty of making the two plates galvanically so nearly equal to each other that a feeble current shall not be produced by the inequality as soon as they are placed in the liquid. Yet neither the polarization of the plates nor their inequality in a galvanic point of view would injuriously affeet the experiments, if these two conditions. did not vary as soon as the liquid is put in motion. But it, appears that such variation really takes place; and therefore the experiments cannot be made by simply sending a galvanic current through the liquid and measuring the intensity, first while the liquid is at rest, and again after it has been set in motion; for, as both the polarization and the galvanic quality. of the plates are altered by the motion of the liquid, the observed difference in the current-intensity may just as well be attributed to this alteration as to a change in the resistance of the liquid.

After putting to the test of practice some proposed methods of observation which need not be here discussed, and finding them not advantageous, I finally adopted the following, which gave good results. (See fig. 1.)

$a b$ is a cylindrical glass tube of uniform diameter and furnished with thres side-tubes $o, d$, and $e$; the distances between $\mathrm{H} 2$ 
$c$ and $d$ and between $d$ and $e$ are as nearly as possible equal. In each of these side-tubes a gold wire is fixed by means of a

Fig. 1.

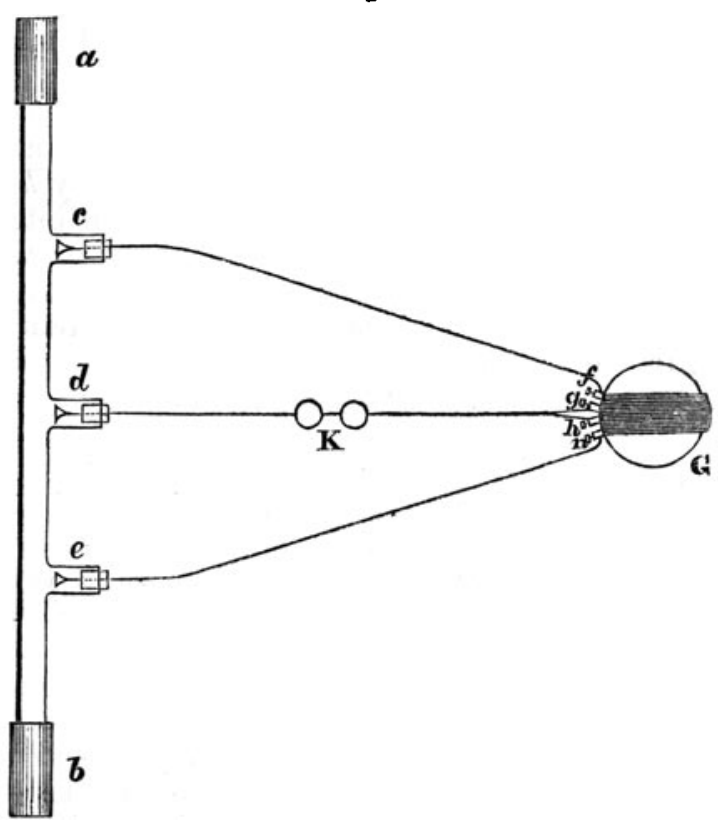

cork and sealing-wax, terminated by a gold disk. On each end of the glass tube a metal cap with a screw-thread is fastened, by which the glass tube can be screwed on to the copper tube through which the liquid to be investigated flows into the glass tube. G represents the magnetometer used in the experiments, the almost perfectly astatic needle-system of which is suspended by a fine silver wire. The position of equilibrium of the needle-system is almost entirely independent of the variations of the earth's magnetic declination-plane, because it is all but exclusively determined by the torsion of the wire. The oscillation-period of the system amounts to about 28 seconds. By a thick copper sheath, which envelops one of the needles, so powerful a damping is produced that the system takes its position of equilibrium after a few oscillations. The readingoff is effected in the usual manner, by aid of telescope and scale. Althongh the magnetometer might possibly have been made more delicate by another arrangement, it possesses the great advantage of an almost invariable position of equilibritim -a circumstance of great value. In the experiments two of 
the positions of the wire of the magnetometer were made use of, in which the resistance and the number of the turns were equal. In the one position the ends of the wire are connected with the binding-screws $f$ and $g$, and in the other with $h$ and $n$ : At $\mathrm{K}$ an electromotor is inserted, one pole of which is connected with $g$ and $h$, and the other with the gold wire in the middle side-tube $d$. The gold wires of the outer side-tubes $c$ and $e$ are united by means of a conducting-wire with $f$ and $n$.

If the glass tube is filled with liquid, and this is at rest, the current from the electromotor divides itself between the two conductions, and these two portions of the current go through the turns of the magnetometer in opposite directions. The resistances of the two conductions are as equal as possible ; and the polarization in the one must be just in the same proportion equal to that in the other, because the polarization of the gold disk in the middle side-tube exerts the same action upon both portions of the current, and the polarization of the two outer polar disks must be of the same nature and amount. Both portions must therefore be nearly the same in quantity, and the sum of their actions on the magnetometer become nearly equal to zero. The experiments also showed that the position of equilibrium of the needle-system differed by only a few scale-divisions from that occupied by the system when no current was passing through the windings of the magnetometer.

If, for instance, the liquid is moving from $a$ to $b$, one portion of the galvanic current goes in the same direction as the flowing liquid, but the other portion goes in the opposite direction against the liquid stream. If the positive pole of the electromotor is connected with the gold disk in the middle side-tube, the stream of liquid and the portion $d e$ of the galvanic current go in the same direction, but the portion $c d$ in the reverse direction to that of the liquid; but if the negative pole of the series is connected with the same gold disk, the conditions are reversed. Now, if the resistance is dependent on the motion of the liquid (as the theory requires), the two portions of the current become unequal, and consequently the needle of the magnetometer must make a deflection, the direction of which can easily be determined beforehand. But there is another reason that the needle of the magnetometer may alter its position of equilibrium : as soon as the liquid is put in motion the polarization of the three gold disks is changed. Yet the change in the polarization of the middle plate has no influence upon the relative intensity of the two parts of the current, because this plate is common to both, and a change in the polarization must act with equal effect on both currents ; it therefore cannot occasion a deflection of the needle. The outer plates do not behave in 
the same manner. Although we try as much as possible to give threm the same magnitude, form, and position, yet a slight difference in this respect may be present; and in consequence of it the variation in polarization effected by the flow of the liquid may be a little greater in one plate than in the other. Under all circumstances a difference between the two plates is present, consisting in the fact that during the flow of the liquid the upper disk is exposed to a greater pressure than the lower one; it is therefore possible that this may cause the needle to make a slight deflection. The deflection which results from variation of polarization, however, we can make as small as we please, by selecting for the experiments liquids the resistance of which is sufficiently great. Moreover it results from the experiments which have been made, that the direction of the deflection is determined exclusively by this-- that the resistance of the liquid becomes less if it flows in the same direction as the galvanic current, but greater if the directions are opposite.

The form of the experiments was as follows:-When the positive pole of the electromotor was connected with the gold disk in the middle side-tube, as soon as the liquid was set moving from $a$ to $b$ I obtained a deflection which indicated that the part of the current passing through the liquid column $d e$ (therefore the portion that had the same direction as the flow of the liquid) possessed a greater intensity than the other. Now the cause of this might either be that the resistance in the column $d e$ became less, and in column $c d$ greater, than when the liquid was at rest, or possibly that by the motion of the liquid the polarization of the polar disk $e$ was rendered feebler than that of the disk $c$. If the change produced in the polarization of the one disk by the motion of the liquid actually differed from that produced in the polarization of the other, this must have depended on the disks in the side-tubes $c$ and $e$ differing somewhat in size, form, and position, or else it was because the one was exposed to a greater pressure than the other. Therefore it cannot be with safety concluded from a single experiment that the resistance to conduction is dependent on the motion of the liquid. Thereupon the glass tube was inverted, so that the liquid flowed from $b$ to $a$, while all the other conditions remained unchanged. The direction of the deflection obtained was the opposite of the previous one, from which it follows that the part of the current which passed through the column of liquid from $d$ to $c$ (and consequently had the same direction as the liquid stream) was more intense than the other. From these two experiments it follows that the difference in form, size, and position that mav have existed 
between the two outer disks, and the alteration of polarization thereon depending, had no effect on the direction of the deflection. If, as in the first experimont, the liquid moved from $a$ to $b$, and the negative instead of the positive pole of the electromotor was connected with the disk in the middle side-tube, the deflection obtained indieated that the part of the current which passed through the liquid column $c d$ (and therefore had the same direction as the flow of the liquid) possessed a greater intensity than the other. In the first two experiments, therefore, the more powerful part of the galvanic current went between the middle polar disk and that one of the two outer disks which was exposed to the less pressure from the flowing liquid; but in the last experiment the more powerful current passed between the middle disk and that one of the two outer disks which received the greater pressure. The difference in the pressures to which the outer disks were exposed during the flow of the liquid cannot, then, have exerted any influence on the direction of the deflection. On the other hand, it appeared in all the experiments that that part of the current which had the same direction as the flow of the liquid was always the intenser ; and for this result no other reason can be given than that the galvanic resistanee becomes less when the current moves in the same direction as the liquid, and greater when the directions are opposite.

In order that the deflections may turn out regular, the liquid employed must exert a great resistance. The importance, in this respect, of the resistance will be seen from the following calculation.

We will denote by $r$ the sum of the principal resistances in the liquid column $c d$, in the conducting-wire $c f$, and in one position of the wire of the magnetometer, and, in a corresponding manner, by $r+\rho$ the sum of the resistances in the liquid column $d e$, in the conducting-wire $e n$, and in the other position of the magnetometer-wire. Because these sums have been made as nearly as possible equal to one another, $\rho$ must be a very small quantity. If we now assume that the negative pole of the electromotor is connected with the gold disk in the middle side-tube, and that the liquid employed is water, this disk cevers itself with hydrogen, the electromotive foree of whose polarization may be called $w$. The two outer disks, on the contrary, become coated with oxygen; and the electromotive force of its polarization in the side-tube $c$ we will denote by $s$, and in the side-tube $e$ by $s+\sigma$. As the polarization is all but perfectly equal in both these disks, the value of $\sigma$ must be inconsiderable. Lot $i$ denote that part of the current which flows through the liquid column $c d$, and $i_{1}$ that part which 
flows through $d e$. The electromotive force of the electromotor we will name $\mathrm{E}$; the principal resistance of it, $R$; and the intensity of the undivided current, J. We have then, by Kirchhoff's laws for the division of the current between several conductors,

and

$$
\begin{aligned}
& \mathrm{E}-(w+s)=\mathrm{RJ}+i r, \\
& \mathrm{E}-(w+s+\sigma)=\mathrm{RJ}+i_{1}(r+\rho),
\end{aligned}
$$

$$
J=i+i_{1} \text {. }
$$

From this we get

and

$$
i_{1}=\frac{\mathrm{E} r-\mathrm{R} \sigma-r(w+s+\sigma)}{2 \mathrm{R} r+r^{2}+\mathrm{R} \rho+r \rho} \quad \text {. . . }
$$

and consequently

$$
i=\frac{\mathrm{E}(r+\rho)+\mathrm{R} \sigma-(r+\rho)(w+s)}{2 \mathrm{R} r+r^{2}+\mathrm{R} \rho+r \rho}, \ldots
$$

$$
i-i_{1}=\frac{\mathrm{E} \rho+2 \mathrm{R} \sigma+r \sigma-\rho w-\rho s}{2 \mathrm{R} r+r^{2}+\mathrm{R} \rho+r \rho} .
$$

The difference $i-i_{1}$ determines the position of equilibrium of the magnetometer-needle when the liquid is at rest. But when the liquid is set in motion, $s$ is changed into $s+\beta, s+\sigma$ into $s+\sigma+\beta_{1}$, and $w$ into $w+\pi$. Denoting the parts of the current which flow through the liquid columns $c d$ and $d e$ respectively by $i_{/ /}$and $i_{/ /}$, we obtain in the same manner

and

$$
i_{\mu}=\frac{\mathrm{E} r+\mathrm{R} \beta-\mathrm{R} \sigma-\mathrm{R} \beta_{,}-r\left(w+\pi+s+\sigma+\beta_{\jmath}\right)}{2 \mathrm{R} r+r^{2}+\mathrm{R} \rho+r \rho} .
$$

$$
i_{/ /}=\frac{\mathrm{E}(r+\rho)+\mathrm{R} \sigma+\mathrm{R} \beta_{1}-\mathrm{R} \beta+(r+\rho)(w+\pi+s+\beta)}{2 \mathrm{R} r+r^{2}+\mathrm{R} \rho+r \rho},
$$

whence $i_{\jmath}-i_{\jmath \prime}=$

$\frac{\mathrm{E} \rho+2 \mathrm{R} \beta,-2 \mathrm{R} \beta+2 \mathrm{R} \sigma+r \sigma+r \beta,-r \beta-\rho(w+\pi+s+\beta)}{2 \mathrm{R} r+r^{2}+\mathrm{R} \rho+r \rho}$.

Subtracting the first difference (4) from the last (7), we obtain the expression for the deflection which arises solely from the fact that the polarization is altered by the motion of the liquid. In this way we get

$$
\left(i_{1 /}-i_{\prime \prime \prime}\right)-\left(i-i_{/}\right)=\frac{2 \mathrm{R}\left(\beta_{1}-\beta\right)+r\left(\beta_{1}-\beta\right)-\rho \pi+\rho \beta}{2 \mathrm{R} r+r^{2}+\mathrm{R}_{\rho}+r \rho} .
$$

The value of $\rho$, however, is so inconsiderable that $\rho \pi, \rho \beta$, $\mathbf{R} \rho$, and $r \rho$ can be neglected. The deflection $u$, which arises only from the alteration of the polarization by the motion of 
the liquid, becomes therefore

$$
u=\frac{\beta_{1}-\beta}{r} . \text {. . . . . . }
$$

This deflection is consequently inversely proportional to the resistance of the liquid; while the deflection occasioned by the change in the resistance is, according to equation (1), nearly independent of the same resistance. If, then, liquids of sufficiently great resistance be selected for the experiments, we can make the variations in the polarization harmless without thereby lessening the deflections produced by the alteration of the resistance. Moreover it follows from equation (1) that the deflections in question are proportional to the cross section of the glass tube and to the velocity of the liquid, but independent of the intensity of the galvanic current employed.

Of the great number of observations conducted in the way above described, it may be sufficient for the purpose before us to communicate only the following.

\section{III.}

For the experiments a cylindrical vessel of thick sheet copper was used, tinned on the inside, and capable of holding about 25 litres. The upper end was hemispherical and provided with three apertures. In one of them a cock was fixed, which was connected with an air-condensing engine by means of a leaden pipe. In order to measure the condensation of the air produced by the engine, a manometer could be screwed on one of the other apertures, which was also made use of for the purpose of filling a part of the vessel with liquid. In the third aperture a copper pipe with a cock was fixed air-tight. That part of this pipe which was inside descended perpendicularly nearly to the bottom of the vessel ; the part outside was bent the form of a semicircle, so that its extremity descended perpendicularly. To this end of the copper tube the above-described glass tube was screwed. The liquid flowing through the glass tube was received in a vessel of glass or wood standing on the floor of the room. Before each experiment, air was forced into the vessel, above the liquid, up to a pressure of two or three atmospheres above the pressure of the external air. No measures were adopted to keep the pressure constant and invariable ; and on this account the velocity of the liquid grew less during the outflow. This inconvenience, however, was of little consequence, because in this investigation the question was rather to obtain an experimental proof of the dependence of the resistance on the motion of the liquid than an accurate determination of the laws of that dependence. 
Experiments with distilled water.

Series 1.-The glass tube had an internal diameter of $5 \cdot 5$ millims.; the distance between the side-tubes was 70 , and the length of the entire tube was 270 millims. The gold disks were drawn so far back in the side-tubes that the distance from the inner surface of the glass tube amounted to 1 centim. Trial was first made whether a deflection could be obtained merely by the motion of the liquid, without inserting an electromotor at $\mathrm{K}$. In this the gold disk in the middle side-tube was connected immediately with the binding-screws $g$ and $h$ by a simple conducting-wire. As was to be expected, in this experiment not a trace of a current was observed.

A series of two Daniell's elements was now inserted at $K$. The rest of the conducting-wires were arranged as shown in fig. 1. The position of equilibrium occupied by the needle when the glass tube was filled with water at rest is denoted by $A$ in the following. As soon as the cock was opened and the water set in motion, the magnetometer-needle made a deflection. The scale-division at which the needle, after a complete deflection, began to return, is given by $\mathrm{B}$; and $\mathrm{D}$ gives the difference between $\mathrm{A}$ and $\mathrm{B}$.

The negative pole of the electromotor was connected with the middle polar disk; the water-flow was from $a$ to $b$ :

$\begin{array}{ccc}\text { A. } & \text { B. } & \text { D. } \\ 617 \cdot 0 & 619 \cdot 0 & 2 \cdot 0 \\ 617 \cdot 0 & 618 \cdot 8 & 1 \cdot 8 \\ 617 \cdot 0 & 619 \cdot 1 & \\ & \text { Mean } . \quad \text {. } & 2 \cdot 2 \\ & & \end{array}$

The deflection shows that the part of the galvanic current which passed from $c$ to $d$ (that is, in the same direction as the water-flow) was more powerful than the other.

The glass tube was now inverted, so that the water flowed through it from $b$ to $a$; every thing beside remained unchanged:

$\begin{array}{ccc}\text { A. } & \text { B. } & \text { D. } \\ 618 \cdot 0 & 616 \cdot 0 & 2 \cdot 0 \\ 618 \cdot 1 & 615 \cdot 9 & 2 \cdot 2 \\ 618 \cdot 0 & 615 \cdot 9 & 2 \cdot 1 \\ & \text { Mean } . \quad . \quad \frac{2 \cdot 1}{}\end{array}$

The deflection (the direction of which was opposite to that of the previous one) indicated that the part of the current which went from $e$ to $d$, and therefore had the same direction as the water-flow, was the more powerful.

The positive pole was connected with the middle disk; the water flowed continuously from $b$ to $a$ : 
dependent on the Motion of the Conductor.

A.

$617 \cdot 0$

$617 \cdot 5$

$617 \cdot 2$
B.

$615 \cdot 8$

$615 \cdot 0$

$614 \cdot 9$
D.

$1 \cdot 2$

$2 \cdot 5$

$2 \cdot 3$

$$
\text { Mean . } \overline{2 \cdot 0}
$$

The deflection had now the same direction as in the next preceding experiment. It thence follows that the part of the current which coincided in direction with the water-flow, going from $d$ to $c$, exerted the greatest action.

The glass tube was then inverted, so that the water flowed again from $a$ to $b$; all beside anchanged:

$\begin{array}{ccc}\text { A. } & \text { B. } & \text { D. } \\ 617 \cdot 0 & 619 \cdot 2 & 2 \cdot 2 \\ 617 \cdot 8 & 620 \cdot 4 & 2 \cdot 6 \\ 618 \cdot 0 & 621 \cdot 0 & \\ & \text { Mean } & \text {. } \quad \frac{2 \cdot 0}{2 \cdot 6}\end{array}$

Consequently it was shown here also that the portion of the current having the same direction as the water-stream was stronger than the other.

Series 2.-This was similar to the preceding, but with the difference that the disks in the side-tubes were not drawn so

- far back, and so were placed near the inmer surface of the glass tube. They were more exposed, therefore, than before to the mechanical action of the flow of water.

The positive pole of the electromotor connected with the middle disk :

$\begin{array}{ccc}\text { A. } & \text { B. } & \text { D. } \\ 628 \cdot 8 & 631 \cdot 8 & 3 \cdot 0 \\ 628 \cdot 8 & 632 \cdot 0 & \\ & \text { Mean } & \mathbf{3 \cdot 2} \\ & & -3 \cdot 1\end{array}$

The direction of the deflection showed that the current in the same direction as the water-flow possessed a greater intensity than the other.

After this the glass tube was inverted, so that the water flowed through it in the opposite direction; all beside unchanged :

A.

$628 \cdot 9$

$628 \cdot 7$

$628 \cdot 9$
B.

$626 \cdot 0$

$625 \cdot 9$

$625 \cdot 2$

Mean . . $\frac{3}{3 \cdot 1}$

The negative pole connected with the middle disk; all else unchanged : 
A.

$631 \cdot 3$

$631 \cdot 5$

$631 \cdot 5$
B.

\begin{tabular}{cr}
$629 \cdot 0$ & $2 \cdot 3$ \\
$629 \cdot 0$ & $2 \cdot 5$ \\
$629 \cdot 0$ & \\
Mean & . . \\
\hline
\end{tabular}

Glass tube inverted; the rest of the arrangement unchanged :

$\begin{array}{ccc}\text { A. } & \text { B. } & \text { D. } \\ 632 \cdot 0 & 634 \cdot 0 & 2 \cdot 0 \\ 631 \cdot 9 & 634 \cdot 0 & 2 \cdot 1 \\ 631.9 & 634 \cdot 2 & 2 \cdot 3 \\ & \text { Mean . . } & 2 \cdot 1\end{array}$

Consequently this series gives the same result as the firstnamely, that the resistance to galvanic conduction is diminished if the conductor moves in the same direction as the galvanic current, but it is increased if the two go in opposite directions.

In all these experiments the velocity of the distilled water was between 9 and 10.5 metres in a second.

Experiments with alcohol containing by volume 24 per cent. water.

With this liquid the experiments were conducted in the same manner as with the distilled water, and led to the same result; it might therefore be superfluous to describe them here.

Experiments with water from the town-supply.

An experiment was made which showed that the resistance of distilled water is about fifty times as great as the water from the conduit. A glass tube in all respects like the one before described was screwed on to the water-pipe in a vertical position. The connexions between the gold disks in the side-tubes, the electromotor, and the magnetometer were arranged in the same way as in the preceding experiments. The only difference with the observations was, that, as the supply of liquid was unlimited, the constant deflections were observed instead of the scale-division at which the magnetometer-needle began to return. By special experiments, in which no electromotor was inserted between the middle disk and the binding-screws $g$ and $h, I$ convinced myself that no galvanic current arose from the water out-flow alone. Therefore no extraneous cause of a galvanic current was present to produce a deflection of the galvanometer-needle.

As the water from the conduit was, in comparison with distilled water, a good conductor, it was to be expected that the polarization of the disks would here exercise a greater influence 
than in the foregoing experiments; this results clearly from equation (9). On this account, the experiments had to be conducted with suitable precautions to prevent the polarization from affecting the direction of the deffections. Before each experiment the electric series had to remain a longer time closed in order that the disks might be completely polarized. During this time the water-cock was 'several times turned on and off. It was only after this had been done that I could reckon upon obtaining constant and regular deflections. It must, besides, be remarked that the galvanic current employed should not be too powerful, because the harmful effect arising from the galvanic difference between the disks increases with the intensity of the current. In the following experiments two Daniell's elements were employed. Out of the observations thus conducted, it will be sufficient to give the two following series. $\mathrm{A}, \mathrm{B}$, and $\mathrm{D}$ have the same signification as before.

Series 3.-Here another glass tube was employed; but the distance of the disks from each other and the diameter of the tube were about the same as in the first tube. The velocity of the water, when the cock was turned full on, amounted to about 10 mètres per second. In reckoning the magnitude of the deflections, the mean was taken of two successive positions of equilibrium with still water.

The negative pole of the electromotor was connected with the middle disk :

$\begin{array}{ccc}\text { A. } & \text { B. } & \text { D. } \\ 608 \cdot 0 & 611 \cdot 0 & 2 \cdot 6 \\ 608 \cdot 8 & 611 \cdot 2 & 2 \cdot 3 \\ 609 \cdot 0 & 610 \cdot 2 & 1 \cdot 2 \\ 609 \cdot 0 & 610 \cdot 5 & 1 \cdot 5 \\ 609 \cdot 0 & 611 \cdot 0 & 2 \cdot 0 \\ 609 \cdot 9 & \text { Mean . . } & \mathbf{1} \cdot 92\end{array}$

The positive pole connected with the middle disk, the rest unchanged :

A.

$626 \cdot 5$

$626 \cdot 5$

$625 \cdot 0$

$626 \cdot 0$

$625 \cdot 5$

$626 \cdot 4$
B.

$628 \cdot 8$

$627 \cdot 5$

$628 \cdot 5$

$627 \cdot 5$

$627 \cdot 2$

Mean . . $\overline{1.98}$

The direction of the deflections indicated in both cases that the resistance is diminished when the galvanic current and the water-flow coincide in direction. 
The glass tube was then inverted, so that the water entered at the other end of it, every thing else remaining unaltered :

$\begin{array}{ccc}\text { A. } & \text { B. } & \text { D. } \\ 627 \cdot 3 & 624 \cdot 9 & 2 \cdot 3 \\ 627 \cdot 0 & 624 \cdot 0 & 3 \cdot 0 \\ 627 \cdot 0 & 624 \cdot 4 & 2 \cdot 6 \\ 627 \cdot 0 & 624 \cdot 0 & 2 \cdot 8 \\ 626 \cdot 5 & 624 \cdot 0 & 2 \cdot 6 \\ 620 \cdot 7 & \text { Mean . . } 2 \cdot 66\end{array}$

The negative pole was connected with the middle disk:

$\begin{array}{ccc}\text { A. } & \text { B. } & \text { D. } \\ 606 \cdot 4 & 605 \cdot 0 & \mathbf{1} \cdot 6 \\ 606 \cdot 8 & 603 \cdot 0 & \mathbf{3} \cdot 9 \\ 607 \cdot 0 & 605 \cdot 4 & 1 \cdot 9 \\ 607 \cdot 5 & 605 \cdot 4 & 1 \cdot 9 \\ 607 \cdot 0 & 604 \cdot 8 & 2 \cdot 2 \\ 607 \cdot 0 & \text { Mean . . } & \mathbf{2 \cdot 3 0}\end{array}$

In both the last two cases, the deflections were (as they should have been) opposite to the preceding ones.

Series 4.-The disks were drawn so far back in the sidetubes that the distance from the inner surface of the glass tube amounted to 8 millims. They were thereby less exposed to the mechanical action of the stream of water.

The negative pole was connected with the middle disk :

$\begin{array}{ccc}\text { A. } & \text { B. } & \text { D. } \\ 626 \cdot 0 & 623 \cdot 0 & 2 \cdot 0 \\ 624 \cdot 0 & 622 \cdot 6 & 2 \cdot 4 \\ 626 \cdot 0 & 622 \cdot 8 & 2 \cdot 7 \\ 625 \cdot 0 & 622 \cdot 5 & 2 \cdot 0 \\ 624 \cdot 0 & 622 \cdot 5 & \mathbf{1 \cdot 5} \\ 624 \cdot 0 & \text { Mean . . } \overline{2 \cdot 12}\end{array}$

The glass tube was inverted, so that the water entered at the other end ; all beside unchanged :

A.

$625 \cdot 5$

$624 \cdot 5$

$624 \cdot 0$

$624 \cdot 5$

$624 \cdot 5$

$624 \cdot 0$
B.

$629 \cdot 0$

$629 \cdot 0$

$629 \cdot 0$

$628 \cdot 5$

$629^{\circ} 0$

Mean . . $\frac{4 \cdot 4}{4 \cdot 42}$

The positive pole was eonnected with the niddle disk, the rest unchanged : 


$\begin{array}{ccc}\text { A. } & \text { B. } & \text { D. } \\ 615 \cdot 0 & 617 \cdot 0 & 1 \cdot 6 \\ 615 \cdot 8 & 616 \cdot 2 & 0 \cdot 8 \\ 615 \cdot 0 & 616 \cdot 2 & 1 \cdot 2 \\ 615 \cdot 0 & 616 \cdot 0 & 1 \cdot 0 \\ 615 \cdot 0 & \text { Mean . . } \frac{1 \cdot 15}{}\end{array}$

After this the glass tube was turned back again; all beside unaltered. The following observations were made a day subsequent to the foregoing :-

$\begin{array}{ccc}\text { A. } & \text { B. } & \text { D. } \\ 612 \cdot 4 & 610 \cdot 5 & 2 \cdot 2 \\ 613 \cdot 0 & 610 \cdot 0 & 3 \cdot 0 \\ 613 \cdot 0 & 610 \cdot 0 & 3 \cdot 0 \\ 613 \cdot 0 & 610 \cdot 0 & 2 \cdot 5 \\ 612 \cdot 0 & 610 \cdot 0 & 2 \cdot 2 \\ 612 \cdot 4 & \text { Mean . . } & 2 \cdot 58\end{array}$

The directions of the deflections in this series were exactly the same as in the previous ones, indicating that the resistance to conduction is lessened when the conductor moves in the same direction as the galvanic current.

In the experiments hero communicated the direction of the deflections is exclusively of importance, and too much weight must not be attributed to their absolute magnitude; for the latter was altered partly by polarization. After the water is set in motion, the two parts into which the galvanic current divides are no longer equal: that which goes in the same direction as the liquid has become greater, while the other is diminished by an equal quantity; and therefore currents of different intensities pass through the disks in the two outer side-tubes. As the polarization, when the currents are as feeble as they were in these experiments, increases with the current-intensity, the more intense part of the current must be somewhat more weakened by the polarization than the other; and since the deflections are proportional to the difference of the two current-intensities, they must be smaller than when no polarization takes place. On the other hand, the polarization of the middle disk, as already remarked, has no effect on the relative quantity of the two parts of the current. Therefore no reliable measure of the change produced in the resistance of the liquid by its motion can be obtained from the experiments made; they show only that such a change actually takes place, and that it cannot fall below the limit which can be calculated from the deflections obtained.

As already stated, we get no deflections, unless an electro- 
motor is inserted between the middle disk and the bindingscrews $g$ and $h$; so that the deflections obtained do not derive their origin from any sort of diaphragm-currents or from any other extraneous cause. The following observation (to which I would direct the attention of those who may desire to control my experiments) does not contradict this:-If, as soon as a series of experiments is concluded, the electromotor be withdrawn and instead of it a simple conducting-wire connect the middle disk with the binding-screws $g$ and $h$, and thereupon the liquid be set in motion, a deflection will be obtained, and this in the same direction as the one a short time before obtained while the electromotor was in the circuit; and if the glass tube be now inverted, wo shall have, quite regularly, a deflection in the opposite direction. It is easy to perceive whence these deflections proceed. When the electromotor was inserted the disks in the side-tubes were polarized, and the two outer ones received a coating of the same sort of gas. Since the disappearance of the polarization is gradual, on this account a polarization-current went through each half of the water column immediately after the removal of the electromotor; but the current in one half was opposite to that in the other, in the same manner as when the electromotor was in the circuit; and when the liquid was put in motion, the resistance in one balf bocame greater, and the other less, than before. Thereby the two currents acquired unequal intensity; and in consequence of this the magnetometer-needle could not but make a deflection.

\section{IV.}

The result of the above experiments I have endeavoured to control by a method of investigation in every respect different from that just described; it was the following :-

Kohlrausch and Nippoldt * have shown that alternating induction-currents, succeeding each other at sufficiently brief intervals, can be advantageously employed for making the resistance of liquids independent of polarization. When water is the electrolytic liquid, only a very thin covering of oxygen can form on the one polar disk, and of hydrogen on the other, during the short time that the current lasts; and in the next instant the directions of the current are reversed, so that the disk which just before was receiving hydrogen receives now oxygen, and vice versâ. If the currents are of equal intensity and short duration and follow close upon one another, it is easy to see that the polarization produced by them must be reduced to a minimum. Indeed the investigations of the above-mentioned philosophers showed also that the polarization was without per* Pogg. Ann. vol. cxxxviii. p. 280. 
ceptible influence on the accuracy of the measurements of the resistance.

We will imagine that the polar disks in the outer side-tubes $c$ and $e$ (fig. 1) are connected with the windings of the magnetometer in such wise that a galvanic current passes through all the windings in the same direction. The middle side-tube is closed, and the disk in it does no service in these experiments. If now an electromotor which emits alternating currents of equal intensity and short duration be inserted between $c$ and $f$, it is evident that the magnetometer-needle must continue in its position of equilibrium if the water in the glass tube is at rest, because the actions of the currents neutralize one another; but when the water is set in motion, then, because this motion alters the resistances, that current which has the same direction as the water is augmented, and the other diminished, and on this account the needle must make a deflection.

In the experiments which were made on the present occasion, induction-currents were not employed, as in Kohlrausch and Nippoldt's investigation, but an arrangement of the following nature. A circular disk of boxwood 75 millims. in diameter, and 20 millims. in thickness is covered on each side with a brass disk. By aid of a winch and cog-wheel the disk can be turned on its axis, making five turns during one revolution of the winch-handle. Fig. 2 represents a portion of the circumference of the disk. From the two brass disks $\mathrm{A}$ and $\mathrm{B}$ little brass plates $a, b, c, d$, and $e$ stand out, all perfectly equal in width, and situated at equal distances one from the other. They are let into the wood so that their surface coincides with the periphery of the disk. Every other of these brass plates is in connexion with the brass disk $A$, and every other in connexion with $B$. The number of these plates is 24 , of which, therefore, 12 are in conducting-union with $A$, and the other 12 with $B$. The distance of the plates from one Fig. 2. another is equal to their width. By a suitable arrangement the brass disk $A$ was connected with one pole of an electromotor, and $\mathrm{B}$ with the other pole. On the periphery of the boxwood disk, two brass springs, tipped with steel, insulated from each other, slide in such a way that they are simultaneously in contact with two neighbouring brass plates. At the same time, therefore, the one is in contact with the plate $a$, and the other with plate $b$. One of these brass springs was connected with the polar disk in the upper side-tube (fig. 1); from the disk in the lower side-tube $e$ a conducting-wire went to one end of the windings of the magnetometer; from the Phil. Mag. S. 5. Vol. 1. No. 2. Feb. 1876. 
other end of the windings a wire conductor went to the other brass spring. From this it is evident that, during the turning of the winch, the direction of the currents was continually alternating, not merely in the glass tube, but also in the coils of the magnetometer. The winch was usually turned once in a second; consequently the currents changed their direction 120 times in the same time.

In order to ascertrin whether, with the alternator just described, the measurements could be carried out quite independently of the polarization, I proceeded as follows :-A parallelepipedal wooden box, asphalted on the inside, was filled with water from the conduit. Two gold plates, just large enough to fill the cross section of the box, were placed at a distance of 50 centims. one from the other. A constant galvanic current was then caused to pass through the water; and as soon as the gold plates were completely polarized, the intensity of the current was measured. After that the plates were placed at a distance of 25 centims., and just so much rheostat wire inserted in the conduction that the intensity was the same as before; the resistance in the water column of 25 centims. length was then equal to that of the rheostat wire employed. I now tried whether by means of the alternating currents of the alternator the same value of the resistance of the water column would be abtained. The wire conductors by which the electromotor, magnetometer, alternator, and water column or the rheostat were connected were so arranged that all the currents flowed through the windings of the magnetometer in the same direction, but were incessantly alternating their direction in the water column or the rheostat. To attain this end, one pole of the electromotor was connected with the brass disk A or B, and the other pole with one end of the windings of the magnetometer. The other end of the magnetometer-wire was joined to the other brass disk B or A. Finally, the two brass sliding springs were united either with the two gold disks or with the ends of the rheostat wire.

When the rheostat was included in the circuit, by employing the alternator a constant deflection of 135 scale-divisions was obtained.

When the water column was inserted, the deflection was 136.5 scale-divisions.

The experiment repeated with the rheostat gave 138 .

The mean of the first and third experiments gives 136.5 scale-divisions, or the same number as the water column.

In another comparison, when the rotation took place with a different velocity, with the water column a deflection of 125.5 , and with the rheostat one of 125 scale-divisions, were obtained. 
It is hence clear that by the alternator the polarization was so completely eliminated that the possibly remaining effect of it fell within the limits of errors of observation.

The gold plates used in these last experiments were many times larger than those little gold disks which were inserted in the side-tubes of the glass tube ; but the intensity of the currents employed was also in nearly the same proportion greater than the intensity employed in the previous experiments; therefore the polarization on the unit of surface of the polar plate cannot have been very different in the two cases. Consequently the proof now given that alternating currents render the polarization harmless must hold good also for the experiments with the small gold disks. Moreover it is self-evident that, for the utility of the method of observation here presented, it is quite immaterial whether the entire polarization is got rid of or not. The only thing requisite is, that both plates shall be in the same condition with respect to polarization; and this requirement is at any rate completely satisfied.

When the water in the glass tube was at rest and the winch of the alternator was turned with a constant velocity, the needle of the magnetometer remained motionless. If the water was then set in motion, I always obtained a deflection which indicated that, of the two alternate currents, that which wont in the same direction as the water was more powerful than the other. These experiments were made both with water from the conduit and with distilled water. The deflections, however, were much smaller than those obtained by the first method. That they would be so could easily be foreseen. Since the distances between the brass plates let into the boxwood disk were equal in magnitude to the width of the plates, the current was closed during only half the time that the winch was moved; consequently the current in one direction did not last longer than a quarter of that time. It must moreover be remarked that, because the alternating currents received an intensity that differed on account of the flowing of the water, polarization was not entirely removed, but somewhat enfeebled the stronger current. These circumstances could not but occasion a diminution of the deflections. Notwithstanding this, the deflections were perfectly distinct and regular. In order to get greater deflections, an ordinary galvanometer, manufactured by Ruhmkorff, with a very astatic needle-system, was provided with a mirror-reading. In this way an instrument was obtained more sensitive than the magnetometor, though at the same time it was affected with the inconvenience that the position of equilibrium of the needle was dependent on the variations in the position of the terrestrial-maguetic declination- 


\section{Prof. J. H. Cotterill on the Distribution of Energy}

plane. The deflections became now considerably greater, and, besides, corresponded perfectly with those obtained with the magnetometer.

The two mutually independent methods of investigation have thus conducted us to the same result, that foretold by the theory -namely, that the resistance to galvanic conduction is diminished when the conductor and the current move in the same direction, but is increased when the directions are opposite.

I will only mention, in conclusion, that I have tried to employ for this investigation a saturated solution of sulphate of copper, with copper poles, and a solution of sulphate of zinc, with amalgamated zinc poles; but these liquids were quite inapplicable, because 1 could never succeed in rendering the polar plates galvanically so equal that, when the plates dipped into the liquid, a slight current would not arise, which immediately varied as soon as the liquid was set in motion. Whence this originated I cannot say. The sulphate-of-zine solution, however, contained some iron.

XIV. On the Distribution of Energy in a mass of Liquid in a state of Steady Motion. By James H. CotTerill, M.A., Professor of Applied Mechanics in the Royal Naval College, Greenwich *.

W HEN a liquid is in a state of steady motion, we know that the total energy of any given particle and of all that follow it in one and the same line of motion is a constant quantity ; or, as we may otherwise express it, the total energy of each elementary stream is uniformly distributed. I am not, however, aware that it has been hitherto noticed that the distribution of energy among the several elementary streams, of which the whole current is supposed to be made up, depends solely on the molecular rotation at each point of the liquid, and is uniform when the motion is irrotational.

For simplicity suppose the motion to be in two dimensions, and to take place either in a vertical or horizontal plane. In the first case the liquid will be under the action of gravity only; and in the second, which will be included in the first, the effect of gravity need not be considered. In the figure, let $A B, C D$ be consecutive lines of motion, and $P Q$ a normal to these lines, and let $P$ and $Q$ be particles moving in these lines." Then, if $h$ be the total bead at $Q$ (that is, the total energy of $Q$ per unit of weight),

$$
h=z+\frac{p}{w}+\frac{v^{2}}{2 g}
$$

* Communicated by the Author. 